\title{
Is fetal CTG a reliable indicator of fetal distress? A prospective study on relationship between CTG suspected fetal distress and immediate postpartum umbilical cord blood pH
}

\author{
Kanika Gupta, Arpana Haritwal, Bela Makhija, Ruchi Bhandari \\ Department of Obstetrics and Gynecology, Max Smart Superspeciality Hospital, Saket, New Delhi, India
}

Received: 2022-01-27.

Accepted: 2022-02-01

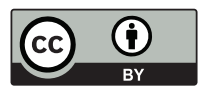

This work is licensed under a Creative Commons Attribution 4.0 International License

J Clin Med Kaz 2022; 19(1):57-64

Corresponding author:

Arpana Haritwal.

E-mail: drarpanaharitwal@gmail.com;

ORCID: 0000-0002-6461-228X

\begin{abstract}
Introduction: Perinatal asphyxia is one of the major causes of neonatal morbidity and mortality. Fetal Cardiotocography (CTG) has been used for long to predict fetal asphyxia. Despite its popularity, it has not been proved to be an ideal tool for monitoring as, although a normal trace is indicative of a normal acid-base status at birth, in about $98 \%$ of cases, an abnormal trace has a low positive predictive value in term of fetal acidosis $(\mathrm{pH}$ less than 7.25).An undisputed evidence of perinatal asphyxia is metabolic acidosis on arterial cord blood or very early neonatal samples: $\mathrm{pH}<7$ and base deficit $>12 \mathrm{mmol} / \mathrm{L}$

Aim: To see the correlation between suspicious/pathological CTG and umbilical cord blood $\mathrm{pH}$ at birth in term pregnancies.

Material and methods: This was a hospital based prospective randomized observational study over a period of 1 year. It was conducted on 165 pregnant women with singleton term pregnancy admitted to labour ward for delivery and having suspicious / pathological CTC trace or meconium stained liquor with normal CTG trace. Immediately after the birth of the neonate, umbilical cord was clamped, cut and umbilical artery cord blood was collected in a pre - heparinized syringe and sent for $\mathrm{pH}$ analysis. Cord blood pH of less than 7.2 was interpreted as acidosis.

Results: The number of acidotic cases (as determined by cord blood $\mathrm{pH}$ less than 7.2) was 2(5.6\%) in normal traces whereas 34 cases (94.4\%) of normal traces were non acidotic. In the suspicious traces, 2 cases (3.2\%) were acidotic and 59 cases (96.8\%) were non acidotic. In the pathological category, 13 cases (19.1\%) were acidotic and 55 cases (80.9\%) were non acidotic. There was no significant association of CTC category with cord blood $\mathrm{pH}$, acidosis, $\mathrm{pO} 2$ or $\mathrm{pCo} 2$ values but that with presence of MSL and grade of MSL was statistically significant.

Conclusion: Abnormal CTG while being a good predictor of the presence of MSL and also the grade of MSL, is a poor predictor of the presence of fetal acidosis and neonatal status after birth. Fetal monitoring using cardiotocography was associated with considerable false positive results. Thus, using fetal heart rate abnormalities alone as a measure of diagnosis of fetal distress during labour is a contributing factor of increasing rate of cesarean sections.
\end{abstract}

Key words: CTG, fetal cardiotocography, fetal distress, cord blood pH

\section{Introduction}

Labour is a stressful event for the fetus. Fetal distress is defined as a condition in which fetal physiology is so altered to make death/ permanent injury a probability within a relatively short period of time and usually considered to denote disruption of normal fetal oxygenation, ranging from mild hypoxia to prolonged fetal asphyxia [1].

Perinatal asphyxia is one of the major causes of neonatal and childhood morbidity and mortality. WHO states that about nine million neonates develop birth 
asphyxia every year and out of these, around one million die, while the same number develop severe consequences like cerebral palsy, epilepsy and delayed developmental milestones.

There are various methods to recognize perinatal asphyxia. The easiest method is intermittent auscultation of fetal heart rate (FHR); but it focuses only on the determination of basal heart rate. Another method is Cardiotocography (CTG). Cardiotocography (sometimes known as electronic fetal monitoring), records changes in the fetal heart rate and their temporal relationship to uterine contractions. In addition to estimating the basal heart rate, it also assesses other parameters like variability, accelerations and decelerations of fetal heart rate [2]. CTG has now become a very popular tool in labour wards. Despite its popularity, it has not been proved to be an ideal tool for monitoring as, although a normal trace is indicative of a normal acid-base status at birth, in about $98 \%$ of cases, an abnormal trace has a low positive predictive value in term of fetal acidosis ( $\mathrm{pH}$ less than 7.25) [3].

Fetal scalp blood sampling gives an accurate intrapartum fetal oxygenation status, but it is invasive, requires expertise, needs relatively large amount of blood, often require to be repeated and is associated with a sampling failure rate of 11$12 \%$ making it relatively less popular [4-6]. One more method is ST analysis (STAN) that involves a combination of the fetal electrocardiogram interpretation and analysis of the fetal electrocardiogram. This analysis requires special machines that are not available widely.

An undisputed evidence of perinatal asphyxia is metabolic acidosis on arterial cord blood or very early neonatal samples: $\mathrm{pH}<7$ and base deficit $>12 \mathrm{mmol} / \mathrm{L}$ [7]. Acute fetal distress induces asphyxia, leading to hypoxia of most of the organs which causes increase in lactic acid level along with alteration in $\mathrm{pH}$ and $\mathrm{HCO} 3$.

Cardiotocography is the most commonly used method to suspect perinatal asphyxia. CTG monitoring is a modern and non-invasive method for assessing both antepartum and intrapartum fetal status. Intrapartum CTG tracings are classified into Normal (category I trace), Suspicious and Pathological trace according to the NICE 2017 guideline [8]:

1. Normal-All features are reassuring

2. Suspicious- 1 non-reassuring AND 2 reassuring features.

3. Pathological-1 abnormal feature OR 2 non-reassuring features.

Aim: To see the correlation between suspicious/ pathological CTG and umbilical cord blood $\mathrm{pH}$ at birth in term pregnancies.

Objectives: Primary: Correlation of suspicious/ pathological CTG or those with a normal CTG but having meconium stained liquor with cord blood $\mathrm{pH}$.

Secondary:

1. Correlation of suspicious/pathological CTG with Apgar at 1 minute and 5 minutes

2. Correlation of suspicious/pathological CTG with meconium stained liquor (MSL)

3. Correlation of suspicious/pathological CTG with cord blood acidosis in Meconium stained liquor cases.

4. Correlation of suspicious/pathological CTG with Neonatal ICU admission, need for resuscitation and convulsions in the first 24 hours after birth.

\section{Material and methods}

The present study included women with term singleton pregnancies in labour with suspicious/pathological CTG tracings or those with a normal CTG but having meconium stained liquor, who were admitted for delivery in the labour ward at a tertiary care hospital and this was correlated with the cord blood $\mathrm{pH}$ immediately at birth. This hospital based prospective randomized observational study was conducted over a period of 1 year (from September 2019 to September 2020)

Intrapartum CTG tracings were taken and classified into Normal (category I trace), Suspicious (category II trace) and Pathological trace (category III trace) according to the NICE 2017 guidelines [8].

Umbilical cord arterial blood was taken immediately after birth, in a pre-heparinized syringe and was sent to the laboratory for $\mathrm{pH}$ study to detect acidosis. A cord blood $\mathrm{pH}$ of less than 7.2 was interpreted as acidosis and a cord blood $\mathrm{pH}$ equal to or more than 7.2 as normal, as described by Saling [9].

\section{Statistical Analysis}

Sample size for the study was calculated as:

$\mathrm{n}=\mathrm{Z} 2 \times(\mathrm{p}) \times(1-\mathrm{p}) / \Delta 2$

Where $\mathrm{n}$ is the sample size,

$\mathrm{Z}$ is confidence interval i.e., 1.96 for $95 \%$

$\Delta$ is confidence level i.e., 0.05 for $\pm 5 \%$

$\mathrm{p}=$ prevalence of the event in the population as determined by previous studies.

Taking incidence of fetal acidosis in term singleton pregnancies as per previous studies as $12.2 \%$ [10] sample size for my study is calculated to be:

$\mathrm{n}=(1.96) 2 \times(0.122) \times(1-0.122) /(0.05) 2=165$

Data was analyzed and statistically evaluated using SPSSPC-20 version.

\section{Inclusion Criteria}

All term (> 37 weeks) singleton pregnancies with vertex presentation in labour with documented suspicious/pathological CTG or Normal CTG with MSL.

\section{Exclusion criteria}

All high-risk pregnancies (anemia, hypertension, thyroid disorders, diabetes, epilepsy, asthma, teenage, elderly)

Intrauterine growth restriction, oligohydramnios, preterm deliveries

Multiple gestation, malpresentations

Abruptio placenta

Fetus with congenital anomalies

Maternal infections and Premature rupture of membranes (PROM)

Patients not giving consent.

\section{Baseline data recording}

All women with term singleton pregnancies in labour with suspicious/pathological CTG and women who may be having a normal CTG but had MSL, fulfilling the inclusion criteria and admitted to our labour ward, were enrolled in the study, after taking an informed consent. On admission, all patients underwent general, systemic and obstetrical examination to rule out any fetal compromise. Labour was monitored by 4-hourly CTG when the cervix was less than $6 \mathrm{~cm}$ dilated and thereafter, continuous CTG monitoring was done. Details were recorded including gestational age at onset of labour, mode of onset of labour (spontaneous or induced), cervical dilatation on admission, membranes status, time of rupture of membranes (spontaneous or artificial), nature/color of liquor after rupture of membranes, CTG tracings, mode of delivery, Apgar score at 1 and 5 minutes, nursery stay, cord blood $\mathrm{pH}$ values, need for resuscitation, occurrence of neonatal seizures were all recorded 
in study proforma sheets. Intrapartum cardiotocography was recorded using Philips Avalon FM20 EF Monitor machine. Tracings were taken by the machine at a speed of $1 \mathrm{~cm} / \mathrm{min}$ for 20 minutes 4 hourly till $6 \mathrm{~cm}$ dilation. Continuous CTG tracings were taken when the patient was in active labour i.e. $>6 \mathrm{~cm}$ dilation. Fetal heart rate patterns were interpreted using the NICE guidelines 2017 [8].

\section{Cord blood collection}

Immediately at birth, possibly before the baby's first breath and before delivery of the placenta, the umbilical cord was clamped at two points, $10 \mathrm{~cm}$ apart, with Kocher's clamps and cut. The umbilical artery was immediately identified in the cord and $5 \mathrm{ml}$ of blood was aspirated with a pre-heparinized syringe. In order to prevent air contact, the syringe tip was sealed with a plastic cover. Cord blood was analyzed by Radiometer ABL800 Basic Machine used at our institute within 30 minutes of collection. Fetal acidosis was assessed using umbilical cord blood $\mathrm{pH}$. An umbilical artery $\mathrm{pH}<7.20$ was defined as fetal acidosis.

\section{Results}

The study included 165 term labouring women who had been admitted in a tertiary care hospital who had either MSL or abnormal CTG and had cord blood ABG taken immediately after delivery of the fetus. $21.8 \%(n=36)$ cases had a normal CTG but with MSL, 41.2\% $(\mathrm{n}=68)$ had a pathological CTG and $37 \%(n=61)$ had a suspicious CTG (Table 1$)$.

Table 1 CTC Category
\begin{tabular}{|l|l|l|}
\hline CTG Category & Frequency & Percentage \\
\hline Normal (but having MSL) & 36 & $21.80 \%$ \\
\hline Pathological & 68 & $41.20 \%$ \\
\hline Suspicious & 61 & $37.00 \%$ \\
\hline Total & 165 & $100 \%$ \\
\hline
\end{tabular}

There was no statistically significant association between maternal age and CTG category (Table 2).

There were 106 primigravidas and 59 multigravidas. The frequency of pathological trace was higher in primigravidas than in multigravidas $(44.3 \%$ vs $35.6 \%$, i.e. 47 cases and 21 cases respectively). However, it was not statistically significant (Table 3).

Similarly, there was no statistically significant association between period of gestation and CTG category (Table 4).

In our study, $21.2 \%$ (35 cases) were induced and $78.8 \%$ (130 cases) had a spontaneous onset of labour. Frequency of pathological trace was similar in both induced and spontaneous labour, 40\% (14 cases) and 41.5\% (54 cases) respectively, making it statistically insignificant (p value- 0.981) (Table 5). There was no statistical association between the mode of onset of labour and CTG category (Table 5).

In our study, 79 (47.9\%) cases had MSL while 86 (52.1\%) cases did not. Out of the 79 cases with MSL, 36 (45.6\%) cases had a normal trace, $21(26.6 \%)$ cases had a pathological trace and $22(27.8 \%)$ cases had a suspicious trace. Out of the 86 cases without MSL, 47 cases $(54.7 \%)$ had a Pathological trace and 39 cases $(45.3 \%)$ had a suspicious trace. The $p$ value for this association between presence of MSL and abnormal CTG category was $<0.001$ for both suspicious and pathological traces, making it statistically significant (Table 6).

Of the $79(47.9 \%)$ cases that had MSL, 19 (24\%) cases had Grade 1 MSL, 32 (40.5\%) cases had Grade 2 MSL and 26
(35.5\%) cases had Grade 3 MSL. 10 (52.6\%) cases of Grade 1 MSL had a normal trace, $3(15.8 \%)$ cases had a pathological trace and $6(31.6 \%)$ cases had a suspicious trace. In cases with Grade 2 MSL, $15(46.9 \%)$ cases had a normal trace, 6 (18.8\%) cases had a pathological trace and $11(34.4 \%)$ cases had a suspicious trace. Amongst the cases with Grade 3 MSL, 11 (39.3\%) cases had a normal trace, $12(42.9 \%)$ cases had a pathological trace and $5(17.9 \%)$ cases had a suspicious trace. The $\mathrm{p}$ value for this association between grade of MSL and abnormal CTG category was $<0.001$ for both suspicious and pathological cases and was statistically significant (Table 7).

There was no statistical association between the Apgar scores at 1 and 5 minutes and abnormal CTG category (Table 8).

The mean $\mathrm{pH}$ for a normal CTG but having MSL was found to be 7.35 with a standard deviation of 0.08 , the mean $\mathrm{pO}_{2}$ was 91.56 with a standard deviation of 12.46 and mean $\mathrm{pCO}_{2}$ was 43.51 with a standard deviation of 9.53 . The mean $\mathrm{pH}$ for a pathological CTG was found to be 7.34 with a standard deviation of 0.1 , the mean $\mathrm{pO}_{2}$ was 88.09 with a standard deviation of 15.29 and mean $\mathrm{pCO}_{2}$ was 45.22 with a standard deviation of 10.15. The mean $\mathrm{pH}$ for a suspicious CTG was found to be 7.36 with a standard deviation of 0.07 , the mean $\mathrm{pO}_{2}$ was 91.43 with a standard deviation of 11.45 and mean $\mathrm{pCO}_{2}$ was 42.89 with a standard deviation of 7.11. All $\mathrm{p}$ values are not significant (Table 9).

The number of acidotic cases (as determined by cord blood $\mathrm{pH}$ less than 7.2) was $2(5.6 \%)$ in normal traces whereas 34 cases $(94.4 \%)$ of normal traces were non acidotic. In the suspicious traces, 2 cases $(3.2 \%)$ were acidotic and 59 cases $(96.8 \%)$ were non acidotic. In the pathological category, 13 cases $(19.1 \%)$ were acidotic and 55 cases $(80.9 \%)$ were non acidotic. There was no significant association of CTG category with cord blood $\mathrm{pH}$, acidosis, and $\mathrm{pO}_{2}$ or $\mathrm{pCO}_{2}$ values (Table 9, Table 10).

Only 5.4\% (9 cases) cases required NICU stay. All of these had acidosis on ABG. In patients who had a normal CTG but had meconium stained liquor $91.7 \%$ (33 cases) did not require any NICU care. Only $8.3 \%$ (3 cases) were admitted to NICU. In patients who had a pathological CTG $92.6 \%$ (63 cases) did not require any NICU care. Only $7.4 \%$ (5 cases) were admitted to NICU. In patients who had a suspicious CTG, 98.4\% (60 cases) did not require any NICU care. Only $1.6 \%$ ( 1 case) were admitted to NICU. The p-value came out to be 0.25 , making the strength of association statistically insignificant (Table 11).

$7.8 \%$ ( 12 cases) required resuscitation. Only $8.3 \%$ cases $(3$ cases) with a normal trace along with MSL required resuscitation and the rest $91.7 \%$ (33 cases) did not. In the pathological trace category, $11.8 \%$ cases ( 8 cases) required resuscitation while the rest $88.2 \%$ (60 cases) did not. Interestingly, only 1 case $(1.6 \%)$ of suspicious trace cases required resuscitation while the rest 60 cases $(98.4 \%)$ did not. The $\mathrm{p}$ value was 0.084 , making the association statistically insignificant (Table 12).

In our study, 3 cases $(1.8 \%)$ had convulsions within the first 24 hours after birth. All of these had acidosis on ABG. Of these, 2 cases had a pathological trace $(2.9 \%$ of pathological cases) and 1 case had a suspicious trace (1.6\% of suspicious traces). None of the normal CTG cases with MSL had convulsions within the first 24 hours after birth. The p value of 0.56 was statistically insignificant (Table 13).

The sensitivity and specificity of a pathological trace was $86.6 \%$ and $38.2 \%$ respectively while that of a suspicious trace was $50 \%$ and $36.5 \%$ respectively. The positive predictive value and negative predictive value of a pathological trace was $19.2 \%$ and $94.5 \%$, while that of a suspicious trace was $3.27 \%$ and $94.5 \%$ respectively. 


\begin{tabular}{|l|l|l|l|l|l|}
\hline \multirow{2}{*}{ Maternal Age } & \multirow{2}{*}{ Total Cases } & \multicolumn{2}{|l|}{ CTG Category } & p value \\
\cline { 3 - 5 } & & Normal (with MSL) & Pathological & Suspicious & \\
\cline { 3 - 5 } & & Frequency (\%) & Frequency (\%) & Frequency (\%) & \\
\hline $20-25$ years & 48 & $07(14.6 \%)$ & $27(56.2 \%)$ & $14(29.1 \%)$ & $0.572-$ not significant \\
\hline $26-30$ years & 63 & $18(28.6 \%)$ & $16(25.4 \%)$ & $29(46 \%)$ & \\
\hline $31-35$ years & 46 & $08(17.4 \%)$ & $23(50 \%)$ & $15(32.6 \%)$ & \\
\hline $36-40$ years & 8 & $03(37.5 \%)$ & $2(25 \%)$ & $3(37.5 \%)$ & \\
\hline Total & 165 & $36(21.8 \%)$ & $68(41.2 \%)$ & $61(37.0 \%)$ & \\
\hline
\end{tabular}

\section{Table 3}

Parity

\begin{tabular}{|l|l|l|l|l|l|}
\hline \multirow{2}{*}{ Obstetric History } & \multirow{2}{*}{ Total Cases } & \multicolumn{2}{|l|}{ CTG Category } & p value \\
\cline { 3 - 5 } & & Normal (with MSL) & Pathological & Suspicious & \\
\cline { 3 - 5 } & & Frequency (\%) & Frequency (\%) & Frequency (\%) & \\
\hline Primigravida & 106 & $20(18.9 \%)$ & $47(44.3 \%)$ & $39(36.8 \%)$ & $0.389-$ not significant \\
\hline Multigravida & 59 & $16(27.1 \%)$ & $21(35.6 \%)$ & $22(37.3 \%)$ & \\
\hline Total & 165 & $36(21.8 \%)$ & $68(41.2 \%)$ & $61(37.0 \%)$ & \\
\hline
\end{tabular}

Table 4 Gestational Age

\begin{tabular}{|l|l|}
\hline Period of Gestation & Total Cases \\
\hline
\end{tabular}

\begin{tabular}{|l|l|}
\hline & Total Cases \\
\hline $37-37+6$ & 21
\end{tabular}

wks

$38-38+6$

wks

$39-39+6$

wks

$40-40+6$

wks

Total

21

48

57

39

39

165

Mode of Onset of Labour

Table 5

Total Cases

\begin{tabular}{|l|l|l|}
\hline \multirow{2}{*}{ Labour type } & Total Cases & CTC \\
\cline { 3 - 3 } & & Nor \\
\cline { 3 - 3 } & & Fre \\
\hline Induced & 35 & 8 \\
\hline Spontaneous & 130 & 28 \\
\hline Total & 165 & 36 \\
\hline
\end{tabular}

CTG Category Normal (with MSL) Frequency (\%) $8(22.9 \%)$

$28(21.5 \%)$

$36(21.8 \%)$

\begin{tabular}{|c|c|c|c|}
\hline \multicolumn{3}{|l|}{ CTG Category } & \multirow[t]{3}{*}{$\mathrm{p}$ value } \\
\hline Normal (with MSL) & Pathological & Suspicious & \\
\hline Frequency (\%) & Frequency (\%) & Frequency (\%) & \\
\hline $8(38.1 \%)$ & $6(28.6 \%)$ & $7(33.3 \%)$ & \multirow[t]{5}{*}{0.254 -not significant } \\
\hline $6(12.5 \%)$ & $23(47.9 \%)$ & 19 (39.6\%) & \\
\hline $11(19.3 \%)$ & $26(45.6 \%)$ & $20(35.1 \%)$ & \\
\hline $11(28.2 \%)$ & $13(33.3 \%)$ & $15(38.5 \%)$ & \\
\hline $36(21.8 \%)$ & $68(41.2 \%)$ & $61(37.0 \%)$ & \\
\hline
\end{tabular}

\begin{tabular}{|c|c|c|c|}
\hline \multicolumn{3}{|l|}{ CTG Category } & \multirow[t]{3}{*}{$\mathrm{p}$ value } \\
\hline Normal (with MSL) & Pathological & Suspicious & \\
\hline Frequency (\%) & Frequency (\%) & Frequency (\%) & \\
\hline $8(38.1 \%)$ & $6(28.6 \%)$ & $7(33.3 \%)$ & \multirow[t]{5}{*}{0.254 -not significant } \\
\hline $6(12.5 \%)$ & $23(47.9 \%)$ & 19 (39.6\%) & \\
\hline $11(19.3 \%)$ & $26(45.6 \%)$ & $20(35.1 \%)$ & \\
\hline $11(28.2 \%)$ & $13(33.3 \%)$ & $15(38.5 \%)$ & \\
\hline $36(21.8 \%)$ & $68(41.2 \%)$ & $61(37.0 \%)$ & \\
\hline
\end{tabular}

\section{Table 6}

Presence of Meconium Stained Liquor

\begin{tabular}{|l|l|l|l|l|l|}
\hline \multirow{2}{*}{ Meconium } & Total Cases & \multicolumn{2}{|l|}{ CTG Category } & p value \\
\cline { 3 - 5 } & & Normal (with MSL) & Pathological & Suspicious & \\
\cline { 3 - 5 } & Frequency (\%) & Frequency (\%) & Frequency (\%) & <0.001 -significant \\
\hline Absent & 86 & $\begin{array}{l}0(0.0 \%) \\
\text { Not included in the } \\
\text { study }\end{array}$ & $47(54.7 \%)$ & $39(45.3 \%)$ & $22(27.8 \%)$ \\
\hline Present & 79 & $36(45.6 \%)$ & $21(26.6 \%)$ & $61(37.0 \%)$ & \\
\hline Total & 165 & $36(21.8 \%)$ & $68(41.2 \%)$ & \\
\end{tabular}

Table 7

Grade of Meconium Stained Liquor

\begin{tabular}{|l|l|l|l|l|l|}
\hline \multirow{2}{*}{ Grade } & \multirow{2}{*}{ Total Cases } & \multicolumn{2}{l|}{ CTG Category } & p value \\
\cline { 3 - 5 } & & Normal (with MSL) & Pathological & Suspicious & \\
\cline { 3 - 5 } & & Frequency (\%) & Frequency (\%) & Frequency (\%) & \\
\hline- & 86 & $0(0.0 \%)$ & $47(54.7 \%)$ & $39(45.3 \%)$ & $<0.001$ - significant \\
\hline 1 & 19 & $10(52.6 \%)$ & $3(15.8 \%)$ & $6(31.6 \%)$ & \\
\hline 2 & 32 & $15(46.9 \%)$ & $6(18.8 \%)$ & $11(34.4 \%)$ & \\
\hline 3 & 28 & $11(39.3 \%)$ & $12(42.9 \%)$ & $5(17.9 \%)$ & \\
\hline Total & 165 & $36(21.8 \%)$ & $68(41.2 \%)$ & $61(37.0 \%)$ & \\
\hline
\end{tabular}




\begin{tabular}{|l|l|l|l|l|}
\hline \multirow{2}{*}{} & \multicolumn{2}{|l|}{} & \multicolumn{2}{|l|}{ PTG Category } \\
\cline { 2 - 5 } & Normal (with MSL) & Pathological & Suspicious \\
\cline { 2 - 5 } & Mean \pm SD & Mean \pm SD & Mean \pm SD \\
\hline APGAR 1 MIN & $8.03 \pm 1.03$ & $7.78 \pm 1.29$ & $8.10 \pm 0.87$ & \\
\hline APGAR 5 MIN & $8.75 \pm 0.60$ & $8.60 \pm 0.98$ & $8.85 \pm 0.57$ & 0.231 \\
\hline
\end{tabular}

Table 9

\begin{tabular}{|l|l|l|l|l|}
\hline \multirow{2}{*}{} & \multicolumn{2}{|l|}{ CTG Category } & S value \\
\cline { 2 - 4 } & Normal (with MSL) & Pathological & Suspicious & \\
\cline { 2 - 4 } & Mean \pm SD & Mean \pm SD & Mean \pm SD & \\
\hline pH & $7.35 \pm 0.08$ & $7.34 \pm 0.1$ & $7.36 \pm 0.07$ & 0.609 \\
\hline p02 & $91.56 \pm 12.46$ & $88.09 \pm 15.29$ & $91.43 \pm 11.45$ & 0.28 \\
\hline pCO2 & $43.51 \pm 9.53$ & $45.22 \pm 10.15$ & $42.89 \pm 7.11$ & 0.321 \\
\hline
\end{tabular}

Table 10

Presence of Acidosis

\begin{tabular}{|l|l|l|l|l|l|}
\hline \multirow{2}{*}{} & \multirow{2}{*}{ Total Cases } & \multicolumn{2}{l|}{ CTG Category } & p value \\
\cline { 3 - 5 } & & Normal (with MSL) & Pathological & Suspicious & \\
\cline { 3 - 5 } & & Frequency (\%) & Frequency (\%) & Frequency (\%) & \\
\hline Acidotic & 17 & $2(05.6 \%)$ & $13(19.1 \%)$ & $2(3.2 \%)$ & $0.0904-$ not significant \\
\hline Non acidotic & 148 & $34(94.4 \%)$ & $55(80.9 \%)$ & $59(96.8 \%)$ & \\
\hline Total & 165 & $36(21.8 \%)$ & $68(41.2 \%)$ & $61(37.0 \%)$ & \\
\hline
\end{tabular}

Table 11

NICU Stay

\begin{tabular}{|l|l|l|l|l|l|}
\hline \multirow{2}{*}{ NICU Stay } & \multirow{2}{*}{ Total Cases } & CTG Category & p value \\
\cline { 3 - 5 } & & Normal (with MSL) & Pathological & Suspicious & \\
\cline { 3 - 5 } & & Frequency (\%) & Frequency (\%) & Frequency (\%) & \\
\hline No & 156 & $33(91.7 \%)$ & $63(92.6 \%)$ & $60(98.4 \%)$ & $0.25-$ not significant \\
\hline Yes & 9 & $3(8.3 \%)$ & $5(7.4 \%)$ & $1(1.6 \%)$ & \\
\hline Total & 165 & $36(21.8 \%)$ & $68(41.2 \%)$ & $61(37.0 \%)$ & \\
\hline
\end{tabular}

Table 12

Need for Fetal Resuscitation

\begin{tabular}{|l|l|l|l|l|l|}
\hline \multirow{2}{*}{ Resuscitation } & \multirow{2}{*}{ Total Cases } & CTG Category & p value \\
\cline { 3 - 5 } & & Normal (with MSL) & Pathological & Suspicious & \\
\cline { 3 - 5 } & & Frequency (\%) & Frequency (\%) & Frequency (\%) & \\
\hline No & 153 & $33(91.7 \%)$ & $60(88.2 \%)$ & $60(98.4 \%)$ & $0.084-$ not significant \\
\hline Yes & 12 & $3(8.3 \%)$ & $8(11.8 \%)$ & $1(1.6 \%)$ & \\
\hline Total & 165 & $36(21.8 \%)$ & $68(41.2 \%)$ & $61(37.0 \%)$ & \\
\hline
\end{tabular}

Table 13

Occurrence of Convulsions within the first 24 hours after Birth

\begin{tabular}{|l|l|l|l|l|l|}
\hline \multirow{2}{*}{ Convulsions } & \multirow{2}{*}{ Total Cases } & \multicolumn{3}{|l|}{ CTG Category } & p value \\
\cline { 3 - 5 } & & Normal (with MSL) & Pathological & Suspicious & \\
\cline { 2 - 5 } & & Frequency (\%) & Frequency (\%) & Frequency (\%) & \\
\hline No & 162 & $36(100 \%)$ & $66(97.1 \%)$ & $60(98.4 \%)$ & 0.56 - not significant \\
\hline Yes & 3 & $0(0.0 \%)$ & $2(2.9 \%)$ & $1(1.6 \%)$ & \\
\hline Total & 165 & $36(21.8 \%)$ & $68(41.2 \%)$ & $61(37.0 \%)$ & \\
\hline
\end{tabular}

Table 14

Sensitivity, Specificity, Positive and Negative predictive value of pathological and suspicious trace

\begin{tabular}{|l|l|l|}
\hline & Pathological & Suspicious \\
\hline Sensitivity & $86.6 \%$ & $50 \%$ \\
\hline Specificity & $38.2 \%$ & $36.5 \%$ \\
\hline Positive predictive value & $19.2 \%$ & $3.27 \%$ \\
\hline Negative predictive value & $94.5 \%$ & $94.5 \%$ \\
\hline
\end{tabular}

\section{Discussion}

The foetus undergoes physiological stress during labour. Fetal morbidity and mortality may occur as a consequence of labour even in low risk patients. Cardiotocography is a simple, non-invasive recordable method of intrapartum fetal monitoring which can be used as a tool to detect hypoxemic events in the foetus-in utero during labour, enabling initiation of appropriate management. We studied the correlation between intrapartum 
CTG in term labouring women with cord blood $\mathrm{pH}$ in their neonates in order to predict acidosis in the foetus and thus to detect the number of cases with actual distress in cases with abnormal CTG traces.

We recruited 165 labouring women who had either a suspicious or pathological CTG, or had a normal CTG but having meconium stained liquor. The study recruited women in the age group of 20 to 40 years. In our study, maximum number of cases were between the ages of 26 to 30 years while the least were between 36 to 40 years. This correlates well with the normal pattern of maximum fertility between 20-30 years of age.

Most cases were between 38 to 38 weeks 6 days of gestation with a decline on either side of this interval. The least number of cases were in the 40 weeks to 40 weeks 6 days period of gestation due to higher rate of spontaneous labour before 40 weeks and also due to most cases that reach 40 weeks of gestation being induced for postdatism in our centre.

The number of cases that went into spontaneous labour were almost four times those that were induced as most cases that were induced due to some medical comorbidity were excluded from our study. Frequency of pathological trace was similar in both induced and spontaneous labour (40\% and $41.5 \%$ respectively), making it statistically insignificant ( $\mathrm{p}$ value0.981).

79 cases were associated with MSL. Out of these, 36 cases (45.6\%) had a normal CTG, 21 cases $(26.6 \%)$ had a pathological CTG and 22 cases (27.8\%) had a suspicious CTG. We also had 86 cases without MSL. A pathological trace was seen in $47(54.7 \%)$ cases of these 86 cases and a suspicious CTG was seen in $39(45.3 \%)$ cases. The association of CTG category with presence of MSL was statistically significant (p-value <0.001). This showed that in the presence of a pathological/suspicious trace, there was a strong possibility of the presence of MSL.

MSL was strongly associated with a pathological trace. It also correlated well with the Grade of MSL. Hence, Grade 1 had more normal traces than Grade 2 and 3 where pathological or suspicious were more. In a study performed by Sunitha $\mathrm{C}$ et al. [11] on 100 women, $4 \%$ of normal traces had the presence of meconium stained liquor and $44 \%$ of abnormal traces had the presence of meconium stained liquor. Similar to our study, they found a positive correlation between the presence of MSL with CTG category. Similar result was obtained in the study conducted Kumar $\mathrm{N}$ et al. [12] in which, out of 30 cases, 5 (16.7\%) had a normal CTG while 10 (33.4\%) had an abnormal CTG, with a positive correlation between CTG category and the presence and grade of MSL.

The mean Apgar score at 1 minute was similar for normal and suspicious traces with slightly lower Apgar for a pathological case. However, the mean Apgar at 5 minutes was similar for all categories of CTG traces. CTG traces did not correlate well with the Apgar score and was a poor predictor of neonatal outcome. This was in contrast to the study by $\mathrm{W}$ M Aboulghar et al. [13], where there was a positive correlation between the Apgar score at 1 and the CTG category, but not with the 5 minute Apgar score. The 1 minute Apgar was less than 4 in $29.2 \%$ cases of pathological traces and $9.6 \%$ of suspicious cases. The 5 minute Apgar score was less than 6 in $8.3 \%$ of pathological and $3.6 \%$ of suspicious traces. In the study by Agrawal SK et al. [14], the mean Apgar scores at 1 minute were $7.5 \pm 0.6$ for normal category CTG and $7.6 \pm 0.3$ for abnormal category CTG. The 5-min Apgar score was $8.8 \pm 0.2$ and $8.7 \pm$ 0.2 respectively. Their result was similar to our study with no significant correlation between CTG category and Apgar score. In the study by van den Berg P et al. [15] on 2659 women, the proportion of infants who had low modified Apgar scores (less than 7) in the group with normal CTG was 8\% (19 of 236) and $0.9 \%$ (22 of 2433 ) for those with abnormal CTG. Similar to our study, there was no statistical correlation with Apgar score and CTG category. In a study by Sowmya D et al. [16], 100 women with either suspicious or pathological traces were recruited. In their study, $25 \%$ of cases in the suspicious category and $38.46 \%$ in the pathological category had 1-minute Apgar less than 7. The 5-minute Apgar was less than 7 in 75\% pathological cases and in $61.53 \%$ suspicious cases. Unlike our study, they showed a positive association between CTG category and Apgar score.

When cord blood ABG characteristics were compared, there was not much difference in $\mathrm{pH}$ for all 3 categories. The pO2 and pCO2 for normal and suspicious category were similar. The pO2 was lower and pCO2 was higher for the pathological category, but this difference was not statistically significant.

The number of acidotic cases were similar for normal and suspicious CTGs. Of all the cases that had a pathological trace, 13 cases (19.1\%) had acidosis ( $\mathrm{pH}$ less than 7.2). Acidosis was present in 2 cases $(3.2 \%)$ of suspicious and 2 cases $(5.6 \%)$ of normal CTG category. There was no statistical significance between CTG category (suspicious/pathological) and presence of acidosis.

The total percentage of acidotic cases was $10.3 \%$ (17 cases) while $89.7 \%$ (148 cases) were non acidotic which is comparable to the study by Kaban et al. [17] (13.26\%) who studied 101 term pregnant women admitted for delivery. In their study 85 neonates had normal cord arterial $\mathrm{pH}$ and 13 had fetal acidosis as diagnosed by cord arterial $\mathrm{pH}$ values less than 7.2. Of the 13 neonates with acidosis, 5 had non-reactive CTG tracings intrapartum. A similar study was performed by Aboulghar et al. [13]. The mean cord blood $\mathrm{pH}$ was $7.24 \pm 0.07$ (range: $7.05-$ 7.39). The acidosis group comprised only $34 \%$ of their neonates, while the rest $66 \%$ had normal cord blood $\mathrm{pH}$. This difference from our study could be attributed to the fact that they included only abnormal CTG cases and did not include any normal cases. Similar to our study, there was no significant association of CTG category with cord blood $\mathrm{pH}$, acidosis, and $\mathrm{pO} 2$ or $\mathrm{pCo} 2$ values. In a study conducted by Kumar $\mathrm{N}$ et al. [12] on 30 women, 13 had abnormal CTG. Out of the 13 women having abnormal CTG results, only $3(23.07 \%)$ showed acidosis (fetal distress in true sense) and required NICU admission. They observed that CTG wasn't a very effective tool for fetal assessment and correlation between cord blood $\mathrm{pH}$ and CTG was not significant.

The sensitivity of a pathological trace for acidosis was good $(86.6 \%)$ but the specificity was poor as it was only $38.2 \%$. The positive predictive value was poor at $19.2 \%$. However, the negative predictive value was good at $94.5 \%$. The sensitivity of a suspicious trace for acidosis was poor at $50 \%$, with an equally poor specificity of $36.5 \%$. The positive predictive value was poor at $3.27 \%$, but the negative predictive value was good at $94.5 \%$. A pathological trace is highly sensitive for detecting fetal acidosis but the specificity for detecting it is poor in our study. However, the presence of a normal trace rules out the presence of fetal acidosis due to a good negative predictive value. A suspicious trace had a poor sensitivity, specificity and positive predictive value, but it had a high negative predictive value like a pathological trace.

These findings are comparable with those found by Parveen et al. [18] who concluded that a normal CTG trace correlates highly with absence of fetal acidosis. Parveen studied 122 cord blood samples using umbilical cord arterial base excess (more than $12 \mathrm{mmol} / \mathrm{l}$ ) at birth to diagnose fetal acidemia. From their study they found that cardiotocography has a sensitivity of 
$15.38 \%$, specificity of $86 \%$, positive predictive value $11.76 \%$ and a negative predictive value $89 \%$. This difference may be because of the fact that they took umbilical artery base excess at birth to diagnose fetal acidosis, while in the present study, umbilical cord arterial $\mathrm{pH}$ value of less than 7.2 was taken to diagnose fetal acidosis.

Steer PJ et al. [19] conducted a prospective study to find correlation among FHR patterns, MSL, umbilical cord arterial blood $\mathrm{pH}$ and Apgar score in 698 cases and found that sensitivity of an abnormal CTG at any time for fetal acidosis (cord arterial $\mathrm{pH}$ less than 7.17 ) was $80 \%$ and for severe acidosis (pH less than 7.08 ) was $83 \%$. However positive predictive value was low: $32 \%$ foetuses had abnormal CTG but no acidosis. If only CTG abnormality in I stage labour was considered sensitivity was $47 \%$ for acidosis \& $67 \%$ for severe acidosis and false positive was $14 \%$. These results were comparable to those found in our study. In a study by Tasnim et al. [1], positive predictive value of CTG was 18\% for fetal hypoxia, 21\% for fetal hypercarbia, $26 \%$ for fetal acidosis and $37 \%$ for base excess. Predictive value of suspicious trace for similar blood indices was 13\%, 13\%, 17\% and $35 \%$ respectively. For pathological trace, predictive value was $50 \%, 83 \%, 100 \%$ and $66 \%$ and respectively. From the findings of this study, although the sensitivity of CTG was found to be low, its high negative predictive value, low cost and ease of carrying out the monitoring supported its use in intrapartum fetal monitoring and in alerting the obstetrician regarding an intrauterine hypoxic event.

CTG abnormality did not correlate well with the need for resuscitation or NICU stay. Again, this showed that it was a poor predictor of adverse fetal outcome.

The presence of a normal CTG ruled out the occurrence of seizures in the first 24 hours after birth but the presence of a suspicious or pathological trace did not necessarily mean that the fetus will have seizures after birth as that was a rare occurrence even with a suspicious or a pathological trace. Similar to our study, the study by W M Aboulghar et al. [13] showed no statistical association between the CTG category and need for NICU stay. In their study, $33.3 \%$ of cases with pathological traces required NICU admission and $23.1 \%$ of those with a suspicious trace required NICU admission.

In a study by Patil SS et al. [20] who performed a similar study on 295 women, there was no association between need for NICU stay, acidosis at birth and CTG category.

In a study by Sowmya D et al. [16], out of 100 women with either suspicious or pathological traces, $34.6 \%$ cases with pathological CTG and 27.08\% with suspicious CTG required NICU admission. This was in contrast to our study and they showed a significant association between the need for NICU stay and CTG category.

In our study, $7.8 \%$ (12 cases) required resuscitation. Only $8.3 \%$ cases (3 cases) with a normal trace along with MSL required resuscitation and the rest $91.7 \%$ (33 cases) did not. In the pathological trace category, $11.8 \%$ cases ( 8 cases) required resuscitation while the rest $88.2 \%$ (60 cases) did not. Interestingly, only 1 case $(1.6 \%)$ of suspicious trace cases required resuscitation while the rest 60 cases $(98.4 \%)$ did not. The $\mathrm{p}$ value was 0.084 , making the association statistically insignificant.

In a study by Patil SS et al. [20] who performed a similar study on 295 women, 24 babies required resuscitation and all 24 of these had severe metabolic acidosis. Like our study, their study showed poor correlation with need for resuscitation.

In our study, 3 cases $(1.8 \%)$ had convulsions within the first 24 hours after birth. All of these had acidosis on ABG. Of these,
2 cases had a pathological trace $(2.9 \%$ of pathological cases) and 1 case had a suspicious trace ( $1.6 \%$ of suspicious traces). None of the normal CTG cases with MSL had convulsions within the first $24 \mathrm{hrs}$ after birth. The $\mathrm{p}$ value of 0.56 was statistically insignificant.

In the study by Patil SS et al. [20], 1.7\% cases had convulsions within the first 24 hours after birth. Similar to our study, this was statistically insignificant.

In a large multicentre study on more than 37000 women by Alfirevic et al. [21], there was a significant association between CTG category and neonatal seizures within the first 24 hours of birth.

\section{Conclusion}

Abnormal CTG is worrisome from both obstetrician's and pediatrician's point of view. Abnormal CTG while being a good predictor of the presence of MSL and also the grade of MSL, is a poor predictor of the presence of fetal acidosis and neonatal status after birth.

Fetal monitoring using cardiotocography was associated with considerable false positive results and subsequent surgical intervention that may have not been necessary. Thus, using fetal heart rate abnormalities alone as a measure of diagnosis of fetal distress during labour is a contributing factor of increasing rate of cesarean sections.

\section{Recommendations}

All patients in active labour should undergo CTG monitoring as although it is not a very specific tool for detecting fetal distress, it's high sensitivity, ease of use and low cost make it a good tool for monitoring.

Decisions for cesarean section based on abnormal CTG findings should be made with caution. Maternal corrective measures as recommended by NICE guidelines should be undertaken first before deciding upon abandoning trial of labour.

In case of an abnormal CTG finding, decision for artificial rupture of membranes should be made wherever feasible to check for the status of liquor and further plan of management should be made accordingly.

Better training in CTG interpretation and its correct interpretation is required to identify, understand and take appropriate corrective measures in those patients that are at a high risk of adverse fetal outcome.

The pediatrician should be kept in the loop in case of any abnormal CTG, as, although the incidence of hypoxia and acidosis is less, the presence of a skilled pediatrician coupled with good resuscitative measures can reduce neonatal morbidity and NICU admissions significantly even in the occasional occurrence of acidosis with abnormal CTG.

Disclosures: There is no conflict of interest for all authors.

Acknowledgements: We thank the postpartum women who participated in the study.

Funding: The financial support for this study was provided by the investigators themselves. 


\section{References}

1. Tasnim N, Mahmud G, Akram S. Predictive accuracy of intrapartum cardiotocography in terms of fetal acid base status at birth. $J$ Coll Physicians Surg Pak. 2009;19(10):632-5. https://doi.org/10.2009/JCPSP.632635

2. Mires G, Williams F, Howie P. Randomised controlled trial of cardiotocography versus Doppler auscultation of fetal heart at admission in labour in low risk obstetric population. BMJ. 2001;322(7300):1457-60; discussion 1460-2. https://doi.org/10.1136/bmj.322.7300.1457

3. Dellinger EH, Boehm FH, Crane MM. Electronic fetal heart rate monitoring: early neonatal outcomes associated with normal rate, fetal stress, and fetal distress. Am J Obstet Gynecol. 2000;182(1 Pt 1):214-20. https://doi.org/10.1016/s0002-9378(00)70515-1

4. Tuffnell D, Haw WL, Wilkinson K. How long does a fetal scalp blood sample take? BJOG. 2006;113(3):332-4. https://doi.org/10.1111/ j.1471-0528.2006.00859.x

5. Wiberg-Itzel E, Lipponer C, Norman M, Herbst A, Prebensen D, Hansson A, Bryngelsson AL, Christoffersson M, Sennström M, Wennerholm UB, Nordström L. Determination of $\mathrm{pH}$ or lactate in fetal scalp blood in management of intrapartum fetal distress: randomised controlled multicentre trial. BMJ. 2008;336(7656):1284-7. https://doi.org/10.1136/bmj.39553.406991.25

6. Westerhuis ME, Moons KG, van Beek E, Bijvoet SM, Drogtrop AP, van Geijn HP, van Lith JM, Mol BW, Nijhuis JG, Oei SG, Porath MM, Rijnders RJ, Schuitemaker NW, van der Tweel I, Visser GH, Willekes C, Kwee A. A randomised clinical trial on cardiotocography plus fetal blood sampling versus cardiotocography plus ST-analysis of the fetal electrocardiogram (STAN) for intrapartum monitoring. BMC Pregnancy Childbirth. 2007;7:13. https://doi.org/10.1186/1471-2393-7-13

7. MacLennan A. A template for defining a causal relation between acute intrapartum events and cerebral palsy: international consensus statement. BMJ. 1999;319(7216):1054-9. https://doi.org/10.1136/bmj.319.7216.1054

8. National Institute for Health and Care Excellence. Intrapartum Care for healthy women and babies Clinical Guideline CG190; 2017.

9. Saling E. Amnioscopy and foetal blood sampling: observations on foetal acidosis. Arch Dis Child. 1966;41(219):472-6. https://doi. org/10.1136/adc.41.219.472

10. Anne Lisbeth Hoffmann, Jesper Ø. Hjortdal, Niels Jørgen Secher, Birgitte Weile, The relationship between Apgar score, umbilical artery $\mathrm{pH}$ and operative delivery for fetal distress in 2778 infants born at term. European Journal of Obstetrics \& Gynecology and Reproductive Biology. 1991; 38(2):97-101. https://doi.org/10.1016/0028-2243(91)90184-M

11. Sunitha C, Rao PS, Prajwal S, Bhat RK. Correlation of intra partum electronic fetal monitoring with neonatal outcome. Int J Reprod Contracept Obstet Gynecol. 2017;6:2174-9. https://doi.org/10.18203/2320-1770.ijrcog20172299

12. Kumar N, Suman A, Sawant K. Relationship between immediate postpartum umbilical cord blood pH and fetal distress. Int J Contemp Pediatr. 2016;3:113-9. https://doi.org/10.18203/2349-3291.ijcp20160141

13. W M Aboulghar, M A Ibrahim, I S Allam, W Hosny, M Otify. Validity Of Cardiotocography In The Diagnosis Of Acute Fetal Hypoxia In Low Resources Settings. The Internet Journal of Gynecology and Obstetrics. 2013; 17(1).

14. Agrawal SK, Doucette F, Gratton R, Richardson B, Gagnon R. Intrapartum computerized fetal heart rate parameters and metabolic acidosis at birth. Obstet Gynecol. 2003;102(4):731-8. https://doi.org/10.1016/s0029-7844(03)00806-8

15. van den Berg P, Schmidt S, Gesche J, Saling E. Fetal distress and the condition of the newborn using cardiotocography and fetal blood analysis during labour. Br J Obstet Gynaecol. 1987;94(1):72-5. https://doi.org/10.1111/j.1471-0528.1987.tb02256.x

16. Sowmya, D., D. Anusha, Vijaya and V. Krishna. Evaluation of Cardiotocography (Ctg) Monitoring For Intrapartum Foetal Surveillance and Its Correlation with Apgar Score. IOSR Journal of Dental and Medical Sciences (IOSR-JDMS). 2018; 17(6):42-50.

17. Kaban A, Cengiz H, Kaban I, Özcan A, Karakaş S. The Success of Cardiotocography in predicting Perinatal Outcome. J Clin Exp Invest. 2012;3(2):168-71. https://doi.org/10.5799/ahinjs.01.2012.02.0137

18. Parveen S. Umbilical cord arterial blood base excess as gold standard for foetal well being screening test validity at term delivery. $J$ Pak Med Assoc. 2010;60(5):347-50.

19. Steer PJ, Eigbe F, Lissauer TJ, Beard RW. Interrelationships among abnormal cardiotocograms in labor, meconium staining of the amniotic fluid, arterial cord blood pH, and Apgar scores. Obstet Gynecol. 1989;74(5):715-21.

20. Patil SS, Sukanya, Rath S, George CE. Study on umbilical cord arterial blood gas analysis and cord blood lactate levels as predictors for adverse neonatal outcome: an observational study. Int J Reprod Contracept Obstet Gynecol. 2018; 7:1494-500. https:/doi. org/10.18203/2320-1770.ijrcog20181342

21. Alfirevic Z, Devane D, Gyte GM. Continuous cardiotocography (CTG) as a form of electronic fetal monitoring (EFM) for fetal assessment during labour. Cochrane Database Syst Rev. 2006;(3):CD006066. https://doi.org/10.1002/14651858.CD006066 